containing media into which disodium $p$-nitrophenylphosphate has been incorporated can be used for streaking, and it has been found practicable to flood inoculated and incubated normal milk agar plates with a small amount of a disodium $p$-nitrophenylphosphate solution. In the latter case, surface colonies of strong phosphatase-producing organisms will turn yellow in a few minutes.

The estimation can be made quantitative by incubating a measured amount of bacterial suspension with a suitably buffered or unbuffered solution of disodium $p$-nitrophenylphosphate for a certain time (half an hour to several hours) and estimating the colour developed in a comparator using permanent colour standards, or photo-electrically. Using such a procedure, a good relationship was found between phosphatase activity and gelatine liquefaction time of Voges-Proskauer positive, methyl red negative Coli-aerogenes organism. Bact. cloacae appears to be in general a non-, or only weak, phosphatase-producer, while Bact. aerogenes and also the $44^{\circ} \mathrm{C}$. positive type (Irregular VI) are strong phosphatase-producers.

In addition to the degree of phosphatase activity and its correlation to another biochemical reaction, there are certain characteristics of the bacterial phosphatase produced which may be of value in differentiating micro-organisms. Pett and Wynne have shown that the $p \mathrm{H}$ optimum may be of value in this respect, and it is suggested that the heat stability in certain media and the activity of a cell-free filtrate may also be of use.

\section{CHEMISTRY AND INTER-RELATIONSHIPS OF ENDOCRINE SYSTEMS AND THEIR PSYCHOLOGICAL EFFECTS}

A evening discussion meeting on this theme was $A$ held on February 26 by the Society for Visiting Scientists at 5 Old Burlington Street, London, W.1, Dr. A. S. Parkes (National Institute for Medical Research, Mill Hill) being in the chair.

Prof. Jean Roche (Collège de France, Paris) spoke first, his subject being the biochemical aspects of hormone actions. One of the major concepts influencing biochemical thought and research in the present century is that of bio-catalysts - substances of great importance profoundly influencing the rate of chemical reactions in the body, and effective in small or minute amounts. Within this general group are substances which were at first thought of as being of a very different nature--vitamins, hormones and enzymes. The demonstration that many, perhaps all, vitamins act by virtue of their intimate association with the enzymes concerned with fundamental metabolic processes in the body is of immense significance.

In the case of the hormones, early work was concerned mainly with their physiological, rather than with their biochemical, actions; the biochemist could approach their study only indirectly by studying their effects on tissue enzymes, and after this action had been shown to be specific, both for the hormone and the enzyme involved. For example, the administration of pestrogens to the castrate female animal produces a marked rise in the alkaline phosphatase content of the uterus, but not of other tissues. Progesterone alone has almost no effect but, given with oestrogens, prevents this rise in enzyme content. Arginase is an enzyme of great importance in the mammals, because of its part in the Krebs cycle for the conversion of ammonia to urea - the main nitrogenous excretion product in this group. More recently, androgens have been shown to increase the concentration of this enzyme in the kidney; the concentration in other organs and of other enzymes in the kidney is not affected. The response is not produced by other sterol hormones without androgenic activity. Similarly, Folley and Greenbaum have demonstrated a marked (hundred-fold) increase in the concentration of this enzyme in the mammary gland following parturition as lactation begins under hormonal influence. These and other observations suggest that tissues can respond by specific enzyme changes in response to specific hormones, and that through such studies the biochemist can make valuable contributions to the least well-known aspect of endocrine function-the mechanism of their peripheral action.

Prof. F. G. Young (Cambridge) suggested that hormones might be defined as substances liberated into the blood stream to produce changes in tissues at a distance. As Prof. Roche had already emphasized, their action is most probably exerted by their effect on the chemical processes of the receptor cells. As a parallel to thoir marked actions in chemically small amounts, the early workers in this field had difficulty in gaining general acceptance for their concepts as there was reluctance to admit that such physically insignificant organs as the pituitary and adrenal glands and the apparently unimportant islet cells of the pancreas could be of supreme importance to the maintenance of life. Full acceptance was delayed until it was shown that the disastrous results of experimental removal of these 'inner secreting' or endocrine glands could be reversed by injections of potent extracts from them. Even the central nervous system, which at the time when the early observations were made, such as that of Addison in 1855, was thought to be the dominant controlling system, has been shown to depend largely on the maintenance of the correct balance of endocrine activity for its proper functioning. The level of sugar in the circulating blood is largely under hormonal control : a doubling leads to diabetes and severe metabolic upset; a halving to coma, convulsions and death.

Chemically, the hormones are a very heterogeneous group ; even in a single gland there is often a multiple secretion rather than a single hormone. Very recently it has been shown that the thyroid, formerly thought to secrete only one effective chemical substance-the iodo-amino-acid thyroxine-probably secretes also the closely related and more active 3'-3-5-tri-iodothyronine.

In conclusion, Prof. Young pointed out that many endocrine glands are derived embryologically from 'barrier' tissues-the ectoderm separating the animal from the external environment, and the endoderm of the alimentary canal coming between the body as a whole and the gut lumen. He postulated that one important action of hormones in the tissues might be concerned with increasing or decreasing the rate at which important metabolites cross the barrier of the cell membranes.

Prof. G. W. Harris (Institute of Psychiatry, University of London) said that the profound effects 
of the endocrines on the nervous system and behaviour, and more recently the influence of the nervous system on endocrine function, have been known in a general way to those engaged in medical practice for many years. The deliberate experimental study of the extent and mechanism of these interrelationships is a more recent development. Of the influence of hormones on behaviour, still very little is known except in certain limited fields. The studies of Beach on the modification of animal behaviour under the influence of sex hormones are outstanding in this field. Nervous control of the endocrine glands has been more extensively studied and more is known of this.

Despite the fact that only two endocrine glands the adrenal medulla and the posterior pituitary-are known to have an anatomically obvious and functionally important direct nerve supply, nervous factors are known to play an important part in endocrine function. Marshall's original investigations on the importance of "exteroceptive factors", such as light and temperature, on the sex rhythms of lower animals, have been confirmed and extended in great detail. More recently the responses of the adrenal cortex under the control of the adrenocorticotrophic hormone of the anterior pituitary have been exhaustively studied. An animal exposed to physical or chemical trauma, or to various procedures such as forced immobilization or loud noises, all presumed to cause mental disturbance and, for want of a more precise term, grouped together as 'emotional stresses', exhibits definite signs of increased adrenal cortical activity. Recent work in Prof. Harris's laboratory seerms to indicate that in rabbits the activity of the thyroid gland, under the control of pituitary thyrotrophic hormone, decreases in response to such 'stresses' that are known to activate the suprarenal gland. The clinical impression that emotional disturbance may be a precipitating factor in the onset: of human hyperthyroidism is another indication of nervous influence on thyroid function.

There is, therefore, a very considerable body of evidence, from many sources, of nervous and mental influence on endocrine function, mediated mainly via variations in the output of the trophic hormones of the anterior pituitary gland.

The discussion at the meeting centred mainly on the influence of psychological factors on endocrine function. Various speakers commented on the difference in response of the experimental animal and man with respect to thyroid function. Prof. Harris replied that the depression seen in his animals was unexpected in view of the information available about human disease. A species difference was postulated by other members of the audience. Different responses in the same species to similar physical stress accompanied by presumably different emotional states were described; in the ritual fighting of male mice kept together, the blood sugar of the aggressor is elevated, but not that of the attacked animal, though both exhibit violent physical activity.

Dr. Parkes, in his summing-up, re-emphasized the importance of the approach discussed by Profs. Roche and Young-the detailed study of the mechanism of peripheral action of the hormones. For the non-chemical endocrinologist, perhaps the most valuable and promising field for future research is the influence that the external environment, acting through the central nervous system, exerts upon the endocrine state of the organism.

\section{ANALYSIS OF MECHANICAL AND ELECTRICAL ENGINEERING FAILURES}

\begin{abstract}
B EFORE the Second World War the British Engine $B$ Boiler and Electrical Insurance Co., Ltd., issued annually a technical report dealing both with original work and with the investigation of engineering failures. Vol. I of a new series has just been published, which more than maintains the high standard of its predecessors*. This publication will be found to be of the very greatest interest by all engineers and metallurgists who are concerned with the field common to the two professions.
\end{abstract}

The first forty-five pages are devoted to a most commendable discussion from the practical point of view of caustic cracking in steam boilers, possibly the most comprehensive treatment which has so far appeared. A very considerable number of cases are discussed in detail, and since neither chemists nor metallurgists are as yet in complete agreement as to the details of the fundamental mechanism involved, and since no reliable means of preventing this dangerous form of deterioration has been developed, these are the more valuable. It is suggested that the best advice that can be offered in the light of present knowledge is that the workmanship of new boilers should be of a high standard, particularly as regards the sealing of the riveted seams on the water side; that care should be taken to avoid over-heating, rapid fluctuations of temperature, and other factors arising under working conditions which may disturb the seams; that special care is required in repair work in connexion with the sealing of such seams; and the maintenance of a minimum caustic alkalinity of the boiler water and the maintenance of a sodium sulphate/sodium hydroxide ratio above $2 \cdot 5$.

A most interesting account by Mr. G. A. Cottell of original work on the sources of error in notched-bar testing concludes this publication. The apparatus is described and the sources of error carefully differentiated; in particular, the influence of the seat between the specimen and the machine has been investigated and a new form suggested which cuts down the loss very considerably and, at the same time, eliminates much of the scatter found under normal conditions. It is shown that the results of such losses may reach as high a value as 25 per cent. It is of more than a little interest that, when using a modified form of specimen or a modified type of test, the yield point is clearly shown, even under notched-bar conditions. A fact of great interest which emerges from this work is the evidence that the speed of fracture exerts a considerable influence on the results. A comparison is made between impact and slow-bend tests on identical specimens, and it is shown that the total work of fracture with the Izod specimen is almost 20 per cent higher in impact than when the specimen is broken slowly. A continuation of this work will be looked for by many.

The remainder of the report is concerned with the examination of twenty-seven cases of service failure, a typical one being that of the brittle fracture of a crane hook, which is referred to strain age-embrittlement in a rimming steel, resulting from the strain and heating due to compression and abrasion. A similar example of strain age-embrittlement in a * British Engine Boiler and Electrical Insurance Co., Ltd. 'Technical Report, New Series, Vol. 1. Pp. 212. (From the Company, 